\title{
Social Environmental Influences on the Development and Resolution of Alcohol Problems
}

\author{
Barbara S. McCrady, Robert A. Zucker, Brooke S. G. Molina, Lyndsay Ammon, \\ Genevieve M. Ames, and Richard Longabaugh
}

\begin{abstract}
This article summarizes the proceedings of a symposium presented at the 2005 Annual Meeting of the Research Society on Alcoholism, Santa Barbara, California, June 25-30. The overall goal of the symposium was to consider the broad impact of the social environment on the development of and successful or unsuccessful resolution of drinking problems. The presentations addressed multiple social environmental influences including: the influence of children on parents (Dr. Zucker), the influence of peers and parents on adolescents (Dr. Molina), the influence of family members on adult drinking (Dr. McCrady), the influence of adult peers/friends (Dr. Kaskutas), and the influence of the occupational environment (Dr. Ames). Dr. Longabaugh, the symposium discussant, addressed models for understanding the relationships between social influences and drinking problems.
\end{abstract}

Key Words: Family, Parents, Spouses, Children, Friends, Occupational Environment.

\begin{abstract}
FAMILY EFFECTS ON ALCOHOLISM STATUS AND ALCOHOLISM RISK OVER TIME: MARITAL PARTNER EFFECTS, PROSPECTIVE FAMILY AND CHILD EFFECTS, AND METHODOLOGICAL CHALLENGES ${ }^{1}$
\end{abstract}

Robert A. Zucker, James A. Cranford, Frank J. Floyd, Bret E. Fuller, and Jennifer M. Jester

\section{Introduction}

When alcoholism diagnosis is evaluated prospectively, autostability is high, suggesting on the one hand that heritable components of the disorder remain active over much of the life course and on the other that the environmental characteristics that sustain high alcohol use are nested with the diagnosis so that attempts at recovery are difficult to sustain. However, diagnostic stability is far from perfect, and a burgeoning literature has indicated that a number of elements in the proximal social environment mediate the stability (Humphreys et al., 1997; McAweeney et al., 2005). Our presentation summarized recent work from a long-term and still

From the Rutgers-The State University of New Jersey, Piscataway, New Jersey (BSM); the University of Michigan, Ann Arbor, Michigan (RAZ); the University of Pittsburgh School of Medicine, Pittsburgh, Pennsylvania (BM); the Alcohol Research Group, Berkeley, California (LA); the Pacific Institute for Research and Evaluation (GMA); and the Brown University, Providence, Rhode Island (RL).

Received for publication November 30, 2005; accepted December 6, 2005.

This study was supported by grant R37 AA07070 from the National Institute on Alcohol Abuse and Alcoholism. Also supported by AA00202 and AA11873 to Molina, DA12414 to Pelham, and AA12342 to Donovan.

Copyright (C) 2006 by the Research Society on Alcoholism.

DOI: 10.1111/j.1530-0277.2006.00080.x

${ }^{1}$ This work was supported by NIAAA Grants R37 AA07065, R01AA12217, and T32 AA07477 to RAZ, and F32 AA05584 to BEF. ongoing prospective family study that examines potential proximal and distal familial mechanisms by which stability and change in alcohol use disorder (AUD) diagnosis and risk for diagnosis among offspring are sustained over time.

\section{Methods}

The Michigan Longitudinal Study (MLS) (Zucker et al., 2000) is a family study of community ascertained male alcoholics, their wives, and their initially 3- to 5-year-old full biological offspring $(n=186)$. An ecologically comparable set of nonsubstance abusing, also initially intact families with young children who were living in the same neighborhoods $(n=120)$ provided a contrast to the alcoholic families. This report draws from: (1) A 3-generational database measuring grandparent and parental AUD, spousal aggression, and aggression to offspring, to predict early and later childhood aggression of third generation offspring (Fuller et al., 2003). Given the importance of aggressive behavior in the etiology of AUD (Zucker and Wong, 2005), this was the proxy indicator we used for later AUD risk. The study involved the construction of crossgenerational structural equation models to model the pathways of aggressive behavior and AUD among grandparents, parents, and children in the MLS. (2) Recent analyses of behavioral interactions among the parents based on a videotaped interaction task where the partners attempted to resolve a problem that caused intense disagreement between them (Floyd et al., in press). (3) A 3-generational database measuring grandparent and parental AUD, spousal aggression, and aggression to offspring, to predict early and later childhood aggression of third-generation offspring (Fuller et al., 2003). The study involved the construction of cross-generational structural 
equation models to model the pathways of aggressive behavior and AUD across the 3 generations. (4) New analyses of retrospective questionnaire data on childhood functioning of the MLS parents.

\section{Results and Discussion}

Marital Assortment as a Homogenizer of the Family Environment. Marital assortment on the basis of alcoholism diagnosis has been reported in the literature for a long time (cf. Windle, 1997), but the features that lead to the assortment have not been delineated. Analyses of MLS parents' baseline data showed what some of those features are: Fathers' and mothers' age of first drunkenness correlated $0.22(p<0.01)$, level of childhood antisocial behavior correlated $0.21(p<0.01)$, and years of education correlated 0.51 $(p<0.001)$. With the possible exception of years of education completed, these other activities were in place before the start of the partner relationships, suggesting that shared values and lifestyle were the initial basis of mate selection, and problem drinking was a part of that profile.

Precursive Family Characteristics That Contribute to Assortment. The presence of these lifestyle characteristics early in the lives of each parent suggests that they are enduring. The Fuller et al. (2003) analyses in fact demonstrated that the stability is not only present developmentally early, but also extends cross-generationally. Thus, utilizing aggressive behavior and childhood conduct problems as proxies for AUD risk, grandparental marital aggression predicted parental childhood conduct problems directly [standardized parameter estimate $(\mathrm{SPE})=0.18$ for mothers and 0.19 for fathers]. The conduct problem indicator, in turn, was one attribute of the assortment.
Differentiations in Marital Interaction as a Function of Parental Alcoholism Subtype and Marital Assortment, or Lack Thereof: Social Interaction Characteristics That Sustain the Assorted Relationship. Despite the considerable heterogeneity known to exist among alcoholics (Babor, 1996), little attention has been paid to the role that heterogeneity of the disorder plays in differentiating the marital environment among subtypes of alcoholics. We compared problem solving interactions among 4 types of alcoholic couples (husband alcoholic with antisocial personality disorder (ASPD) or not, paired with alcoholic or nonalcoholic wives) and compared their interactions patterns with each other and with a both-spouses-nonalcoholic group (Floyd et al., in press). Consistent with a hypothesis of differentiated assortment, couples with an antisocial alcoholic husband had higher levels of hostile behavior, regardless of wives' alcoholism status (Fig. 1). Consistent with a drinking partnership (concordance/discrepancy) hypothesis (cf. Leonard and Roberts, 1998), rates of positive behaviors and the ratio of positive to negative behaviors were greatest among couples in which either both or neither of the spouses had alcoholic diagnoses (i.e., where spouses were diagnosis-concordant) and were lowest among alcoholic husbands with nonalcoholic wives (where spouses were diagnosis discordant) (Fig. 2). Previous work has shown that the P:N ratio is important for the maintenance of marital stability (Gottman, 1994). These findings suggest that one possible mechanism whereby discrepant drinking within couples leads to marital distress is through lower ratios of positive to negative behaviors.

Autostability of Conduct Problems and Marital Conflict as a Stabilizer of Adult Diagnosis and Also of Child Risk Over Time. The Fuller et al. (2003) analyses also showed

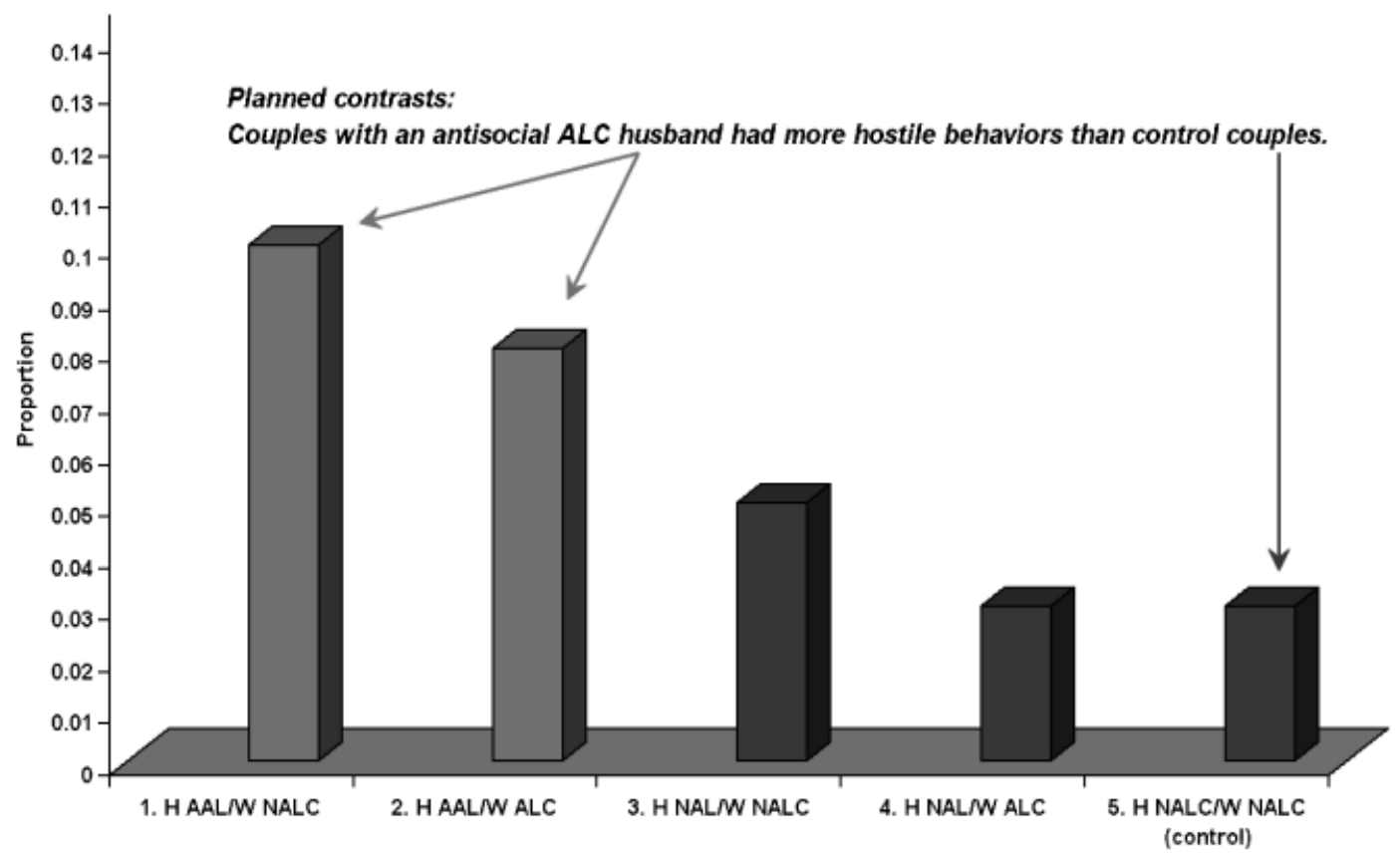

Fig. 1. Mean levels of hostile behavior as a function of couple type. 


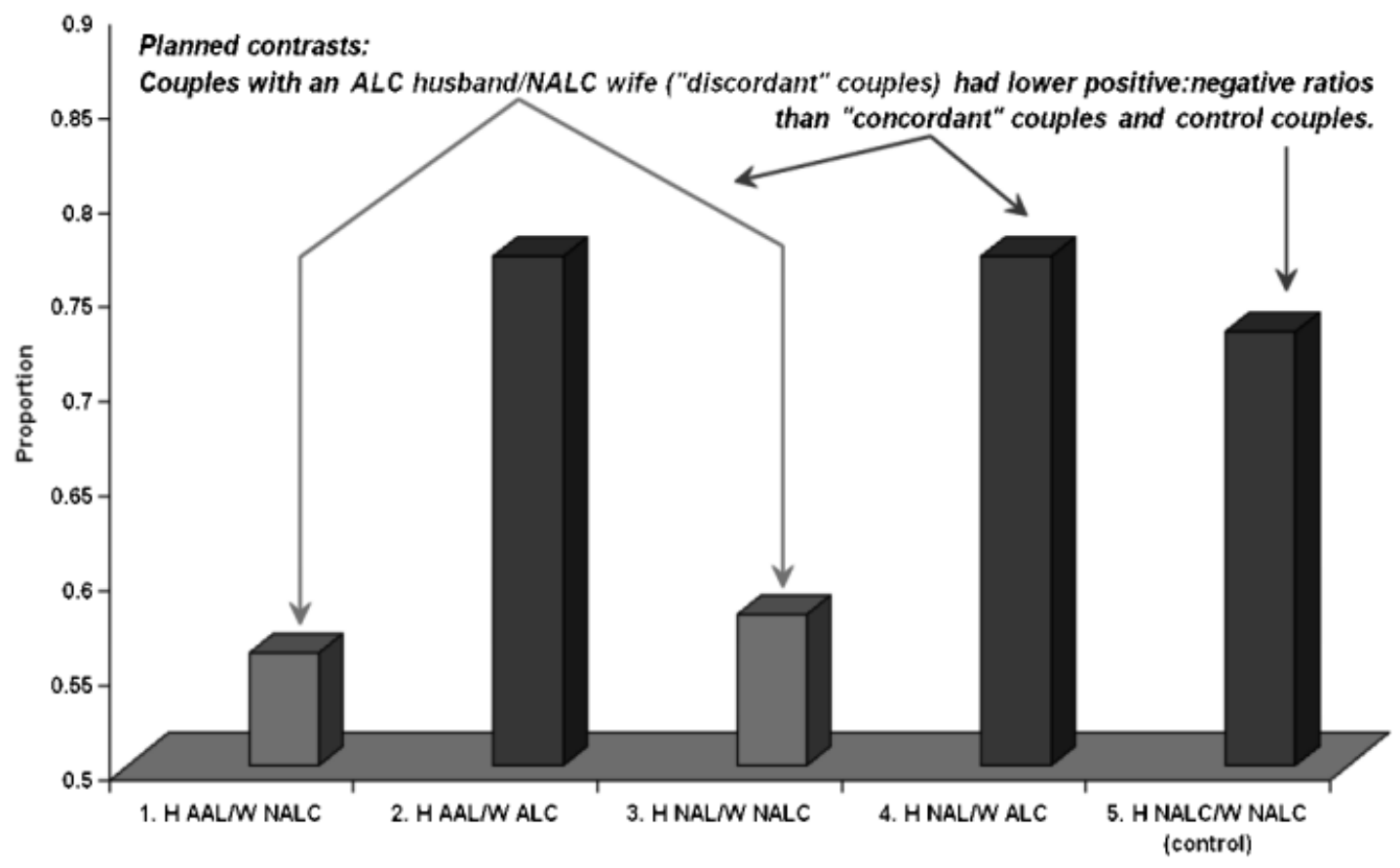

Fig. 2. Means scores on positive:negative ratio as a function of couple type.

significant continuity (autostability) of the assortment characteristics over time, which in turn predicted stability of marital conflict over time and continuity of parental AUD diagnosis as well as continuity of child AUD risk. Thus, the parental childhood conduct problem assortment indicator predicted (1) level of marital conflict at early marriage (when offspring were 3-5 years of age) (SPE $=0.31$ for mothers and 0.27 for fathers) and (2) marital conflict early in marriage in turn predicted marital conflict 6 years later ( $\mathrm{SPE}=0.37$ for mothers and 0.40 for fathers). (3) The parent childhood conduct problem indicator also independently predicted parental AUD diagnosis in early marriage ( $\mathrm{SPE}=0.37$ for both mothers and fathers).

Social Environmental Transmission of AUD Risk From Child to Parent to Child. Child aggressiveness in preschool predicted parent to child aggression 6 years later (SPE of early child aggressiveness to later parent to child aggression was 0.18 for both parents); in turn level of parent to child aggression at age 9 to 11 predicted level of child AUD risk at 9 to 11 (SPE $=0.23$ for mothers and 0.16 for fathers).

Potential Confounds and Methodological Challenges. The 3 generational networks of relationships existing here indicate that the manner in which family relationships create proximal and distal effects involves substantial acrossgenerational relationships from grandparent to parent to child which sustain continuity of risk. The pattern of relationships suggests substantial genetic continuity, niche seeking (relationship assortment), as well as social transmission of risk through the modeling and learning of aggressive behavior. The analytic dilemma is that in the behavioral models so many of us work with, including the ones presented here, social environmental risk is confounded with indirect genetic effects. Under this scenario, putative socialization effects of parent behavior upon child behavior are in fact the common display of genetic as well as phenotypic similarity and are transmitted at conception rather than through learning. Family designs, involving both parents and children, and the availability of genotyping for both generations, will ultimately allow these effects to be disentangled. This is an essential task, given the heavily nested, assorted set of relationships among risk variables that is present in high-risk families.

\section{INTERPERSONALLY AND ENVIRONMENTALLY MEDIATED RISK FOR ALCOHOL PROBLEMS AMONG ADOLESCENTS WITH CHILDHOOD ATTENTION- DEFICIT/HYPERACTIVITY DISORDER}

Brooke S. G. Molina, W. E. Pelham, Jr., Michael P. Marshal, Elizabeth M. Gnagy, and John E. Donovan

Children with attention-deficit/hyperactivity disorder (ADHD) are vulnerable to the early use and abuse of alcohol because of the core symptoms of the disorder (inattention, impulsivity, and hyperactivity) and because of the associated features, functional impairments, and other risk factors for alcoholism common in this population (Molina and Pelham, 2003; Pelham et al., 1998a). Indeed, models of alcoholism etiology (e.g., Sher, 1991; Sher and Trull, 1994) include dispositional characteristics synonymous with ADHD features as key variables that mediate the deleterious effects of parental alcoholism on the later development of offspring drinking. Etiologic 
models such as these also include interpersonal influences with the potential to further explain vulnerability through mediating and moderating processes. This presentation described preliminary findings from the Pittsburgh ADHD Longitudinal Study (PALS) that illustrate the potential for both deleterious and beneficial interpersonal (parental and peer) processes in the development of drinking among youth with childhood ADHD. Thus, we examined the influence of parental alcohol use (both self-reported and as perceived by probands), deviant peer affiliation, adolescent expectancies, and parenting practices on the development of alcohol use in samples of ADHD individuals followed from childhood and a comparison group of non-ADHD participants.

The PALS is a longitudinal study of 364 children diagnosed with ADHD at the ADD (attention deficit disorder) Program, Western Psychiatric Institute and Clinic, between the years of 1987 and 1996 and 240 demographically similar individuals without ADHD. Follow-up of the probands was initiated an average of 8.35 years after diagnosis and participation in an 8 week summer treatment program for ADHD (Pelham and Hoza, 1996). Diagnosis was based on a standardized diagnostic maternal interview with $\mathrm{PhD}$ level clinicians including widely used parent and teacher behavior rating scales (e.g., Pelham et al., 1992). The summer program included behavior management, parent training, medication trials, and skills training in academics, athletics, and social interaction. At the first PALS follow-up, the probands ranged in age from 11 to 28 years old (participation rate, $70.5 \%$ ).

When the proband follow-up was initiated (same adolescent to young adult age range) the 240 individuals without ADHD ("controls") were recruited from pediatric practices that overlap with the probands' pediatricians and from local advertisements. Controls were screened by phone to be free of a history of ADHD diagnosis or ADHD symptoms sufficient in number that a diagnosis was probable in the lifetime. Parents, in addition to young adults, provided these reports. Probands and controls were matched as a group on age, gender (for ADHD, $89.6 \%$ male; for non-ADHD, $88.7 \%$ male), and other demographic characteristics. Additional details of sample recruitment are available (Faden et al., 2004; Molina et al., under review).

For this symposium, the current presentation focused on interpersonal processes with the potential to explain and moderate risk for early episodic heavy drinking among the adolescents in the PALS ( $n=163$ probands; $n=120$ controls, aged 11-17 years). Data also are presented from an earlier adolescent follow-up of children from the same recruitment source (142 probands and 100 controls; see, Molina and Pelham, 2003).

Heavy drinking in adolescence is a well-established marker of vulnerability to chronic alcohol use and interpersonal influences on its development include parent- and peer-mediated effects such as parental alcoholism (Chassin et al., 2004) and the proximal impact of affiliation with peers who use and tolerate the use of drugs and alcohol (Marshal et al., 2003). Because parental AUDs have been linked to offspring behavior problems that include ADHD features and comorbidities, parental alcohol disorder and alcohol use may be important determinants of early heavy drinking among youth with ADHD. Moreover, impaired social functioning among youth with ADHD has long been a well-established clinical problem (Pelham and Bender, 1982), and persistence into adolescence has been documented (Bagwell et al., 2001). To the extent that difficulty in this arena drives youth with ADHD to select or to be befriended by deviant peers, peer influence effects may also help to explain early and heavy drinking among youth with ADHD.

The deviance-proneness pathway to problem drinking emphasizes the importance of socialization influences that include affiliation with peers who model or otherwise facilitate alcohol consumption. This proposed pathway, however, also includes dispositional variables characterized by impulsivity and behavioral disinhibition that facilitate deviant peer affiliation, especially in the context of deficient parenting (for reviews, see Sher, 1991; Sher and Trull, 1994). Thus, children with ADHD should theoretically have elevated vulnerability to these socialization effects given their core deficiencies in cognitive and behavioral control. Indeed, in our earlier follow-up of 142 proband adolescents, we found that the association between deviant peer affiliation, measured as adolescent report of friends who use and tolerate the use of alcohol and other drugs, and heavy alcohol use (frequency of drunkenness and 5 or more drinks at a time) was significantly stronger for the probands than for the controls (Marshal et al., 2003). Although this study was limited to cross-sectional examination of the dependent measures, it provides preliminary support for the hypothesis that peermediated drinking is an important aspect of early (i.e., adolescent) alcoholism vulnerability in this population. We are currently examining these processes longitudinally in the PALS dataset.

Parent alcoholism may reflect a number of biopsychosocial risk processes for offspring alcoholism (Sher, 1991) that are relevant to youth with ADHD. Accordingly, we found a 2-fold elevation in rates of alcoholism among the mothers [odds ratio $(\mathrm{OR})=2.69$ ] and fathers $(\mathrm{OR}=2.39)$ of the probands compared with the parents of the controls. These preliminary data, based on self-report (SCID-NP) or spouse report (MAST-S) from Wave 1, are important because they reveal a widely accepted risk factor for alcoholism in a group of children carefully diagnosed with ADHD. Parental alcoholism, however, did not predict proband or control heavy drinking at Wave 2. In future analyses, we will examine whether this failure to predict is due to our analytic approach (future analyses will use growth modeling to consider individual variation in rate of change in drinking over multiple waves of data), possible 
interactions in the data with ADHD status or other comorbidities (i.e., antisocial comorbidities), or our selection of predictor (e.g., parental alcoholism diagnosis vs parental heavy drinking).

For example, an important aspect of socialization models of drinking is that perceptions of drinking by others and their reasons for drinking partly drive behavior. For example, cognitions regarding alcohol develop at a young age before the onset of drinking, and beliefs regarding the anticipated effects of alcohol prospectively predict later drinking. In the PALS data, we found that adolescent-perceived frequency and quantity of drinking by their parents predicted heavy drinking 1 year later, even after controlling for Wave 1 drinking. Furthermore, these effects were significantly mediated by adolescents' positive alcohol expectancies, mediation $z=2.60$, for probands and for controls. Thus, although alternative explanations are possible, one interpretation of these findings is that adolescent-perceived parental drinking (which did not differ between probands and controls) partially drives the development of cognitions that correlate with drinking behavior. Whether drinking that emerges from this pathway persists into early adulthood and later is an important question for future analyses. Interestingly, we found that negative alcohol expectancies predicted less heavy drinking 1 year later for controls, but not for probands, suggesting the possibility that a self-control or restraint capacity may exist for controls that is not available to probands. Other findings of ours, that coping skills are stronger among control than among proband adolescents, are suggestive of this possibility (Molina et al., 2005).

Finally, as further evidence of the importance of socialization processes that may affect drinking among youth with ADHD, we found that more adolescent-reported monitoring by parents at Wave 1 predicted less frequent heavy drinking 1 year later, after controlling for Wave 1 drinking. This effect did not differ for probands and controls, suggesting that parenting efforts in this high-risk group may be just as beneficial for youth with ADHD as for youth without this vulnerability. This finding, which is commonly reported in other types of samples (e.g., Dishion and McMahon, 1988), has clinical significance because of implications for treatment. Psychostimulant medication is currently the treatment of choice for ADHD among many practitioners, but our findings indicate the importance of continuing to research additional or complementary strategies that may aid in the reduction or prevention of drinking (Pelham et al., 1998b). In addition, our findings suggest the importance of continuing to examine different aspects of the parent-child relationship that may affect drinking trajectories over time and the eventual continuation or resolution in adulthood.

Taken together, our findings provide a preliminary investigation into interpersonal processes that may affect the initiation of heavy drinking in a high-risk group, children with ADHD. Future analyses will expand on these results by adding multiple waves of data to separate youth with ongoing versus "developmentally limited" drinking trajectories and to consider the interplay among these processes as they lead to, or protect from, problem drinking.

\section{THE RELATIVE INFLUENCE OF DIFFERENT FAMILY MEMBERS ON WOMEN'S RESOLUTION OF THEIR DRINKING PROBLEMS ${ }^{2}$}

\section{Barbara S. McCrady and Elizabeth Epstein}

\section{Introduction}

Families have an important influence on drinkers at all stages of change. Data on the influence of families on problem recognition come from a national epidemiological study of drinking in the United States (Room et al., 1991). They reported on family members' responses to individuals who had met at least 2 criteria for an AUD. They found that mothers were most likely to have either commented on or suggested a reduction in drinking $(43 \%)$, followed by spouses $(38 \%)$, friends $(26 \%)$, fathers $(24 \%)$, siblings $(21 \%)$, and children $(12 \%)$. In the help-seeking phase, studies with clinical populations suggest that family influence on help-seeking may differ for men and women with AUDs. For example, Beckman and Amaro (1986) reported that men are encouraged by their wives to seek help, whereas women are discouraged by their husbands but encouraged by their mothers, siblings, and children. Family involvement in treatment has been associated with more positive treatment outcomes in a variety of alcoholdependent populations (reviewed in McCrady, 2004), and data suggest that network support for drinking from family, the workplace, or the network as a whole is associated with poorer outcomes (Longabaugh et al., 1998).

Longabaugh and Beattie (1985) proposed a model to explain the relationships between social influences and drinking outcomes, suggesting that these relationships are mediated by the degree to which the individual has a higher level of investment in the social network. Further, Longabaugh and Beattie discriminated between general social support and social support specific to drinking. Some of their later research provided empirical support for their proposed model (e.g., Longabaugh et al., 1993).

The presentation had 3 major goals: (1) to describe the social networks of treatment-seeking alcohol-dependent women, (2) to examine the influence of different types of family members on women's response to treatment, and (3) to test Longabaugh and Beattie's model of social investment as a moderator of the relation between social network behavior and drinking outcomes.

\section{Methods}

Data for the presentation were drawn from a 5-year, randomized clinical trial comparing 20 session individual

\footnotetext{
${ }^{2}$ This work was supported by NIAAA Grant R37 AA07070 to BSM.
} 
and conjoint cognitive-behavioral therapies for women with AUDs. The recruited sample included 102 women with alcohol abuse/dependence and their male partners. The women were middle-aged, most were Caucasian $(95 \%)$, and most $(89 \%)$ were married. About half the women were employed full- or part-time, and median household income was close to $\$ 90,000$. The women were frequent, heavy drinkers before treatment, drinking on two-thirds of the days in the 3-month pretreatment baseline, averaging about 8 standard drinks per drinking day.

To test study questions, 2 major measures were used - the Timeline Followback Interview (TLFB) and the Important People Interview (IPI, Longabaugh et al., 1998). The primary variable used from the TLFB was percent days abstinent. From the IPI, 2 sets of variables were derived. Measures of social network function included: (1) acceptance or encouragement of drinking (No. of family members), (2) not accepting drinking or leaving/making client leave when she was drinking (No. of family members), and (3) mean spouse encouragement of not drinking. Measures of social investment included: (1) sizes of the family origin and the nuclear family included and (2) perceived importance of the family.

\section{Results and Discussion}

Women described fairly diverse social networks. They were most likely to list their children $(56 \%)$, mothers $(52 \%)$, and sisters $(41 \%)$ as part of their social networks. Fathers $(18 \%)$ and brothers $(11 \%)$ were listed much less often. Women also were asked to designate the 6 most important persons they listed as part of their social network. Among women who listed a child in the social network 91\% also listed the child as "important"; comparable figures were $75 \%$ for listed mothers, $71 \%$ for listed sisters, $61 \%$ for listed fathers, and $36 \%$ for listed brothers. Twothirds of the individuals in the women's social networks were drinkers, 1 in 9 were described as heavy drinkers, and 2 in 9 were described as abstainers.

After describing the social networks, we then tested family variables as predictors of drinking outcomes, using percent abstinent days (PDA) as the dependent variable for all analyses and looking separately at outcomes for the first and second 6 months after the scheduled end of treatment. For all analyses, baseline PDA, age, and treatment condition were entered as control variables. Two separate regressions were run to model network support and network composition as predictors of drinking outcomes. For both analyses, positive and negative network support for drinking were tested, using the number of family encouraging drinking, the number of family not accepting drinking, and spouse encouragement for not drinking as the network support variables. In the first regression, network composition was modeled as the types of family members the woman listed in her social network, including the number of members of the family of origin and the number of members of the nuclear family in the social network. In the second regression, network composition was modeled as the types of family members the woman listed in her social network, including the presence of the mother, sister, and children. Three family variables predicted PDA 6 months after treatment - the number of family not accepting drinking, the number of members of the family of origin listed in the social network, and listed a sister in the woman's social network. At 12 month posttreatment, network composition continued to predict PDA, but family reactions to drinking no longer predicted PDA.

In the last set of analyses, we modeled social investment as a moderator of the relation between family responses to drinking, network composition, and PDA. We used 2 variables as markers of social investment - the number of family listed in the social network and the perceived importance of the family. We tested social investment variables as moderators by examining the interaction between the social investment variable and family response to drinking. Interactions between perceived importance of the family and family responses to drinking were not significant. However, the interaction between family size and the number of family not accepting drinking did predict PDA both 6 and 12 months after treatment.

In summary, children, mothers, and sisters in the network were cited most often and most often perceived as important; male relatives were less often cited and of less perceived importance even when cited. In our analyses, however, we did not control for whether the women had parents, children, or siblings, so the variables represent raw numbers rather than the proportion in each category of family member that the woman included in her list of important people. We also found that family composition, particularly listing more members of the family of origin and including a sister in the social network, predicted women's abstinence throughout follow-up. In particular, we found that having more family members who did not accept abstinence or who actually physically distanced themselves from the woman when drinking predicted poorer outcomes. It may be that the negative behavior of family members leads to greater stress or negative affect for the woman, which does not facilitate success. However, it also may be that the families of women with the most severe or chronic AUDs are those who are reacting most negatively. As we did not control for severity of the AUD in these analyses, it is unclear which interpretation is more appropriate.

A surprising finding was that the male partner's behavior was not predictive of outcomes in this sample. However, the sample was of alcohol-dependent women whose male partner was willing to engage in conjoint treatment, and in our sample the likelihood that the men were seen as encouraging the women to drink was extremely low, so the nature of the sample may explain these results. We also know that the male partners changed their behavior considerably during treatment, so pretreatment measures of spouse behavior may be less meaningful than pretreatment measures of the actions of other family members in this sample. 
Finally, we examined 2 measures of social investment to see if either interacted with family behavior to predict outcome and found that the size of the family of origin was a significant moderator, but the perceived importance of the family was not a significant moderator. Overall, the significant interaction between network size and network behavior may be interpreted as support for Longabaugh and Beattie's model. Listing a large number of family members on the IPA may mark the woman's high social investment in her family. When social investment in the family is high, then having more family members who do not accept the drinking positively impacts the woman. However, if social investment in the family is low, then family members' negative reactions have a negative impact on the women - perhaps leading her to feel angry and resentful and therefore drinking more during follow-up. There are, however, other possible explanations of the interaction effect. For example, it may be that if some family members set limits the impact on the woman's drinking is positive in a large family because even as the woman experiences negative feedback from some family members, other family members still are providing positive support for change, so she experiences the best of bothhonest feedback and support for change. However, it may be that the number of family members listed on the IPA is reflective of actual family size and that small, rejecting families are a particularly lethal combination.

The results suggest the salience of several factors to consider in treatment of women with alcohol dependence. First, clinicians should consider the size of female clients' family of origin and the kind of contact they have with them. Involvement in treatment of the family of origin may be important, particularly understanding how family members have reacted to drinking, and clinicians may want to find ways to buffer negative reactions of family members, particularly for women with few family members who are a significant part of their lives. A second and particularly interesting finding was the importance of having a sister who is important to the woman. The data provide no hints to the ways in which a sister contributes to the woman's successful abstinence, and further research should look at the relative contribution of sisters to providing tangible, emotional, and/or guidance support as the woman attempts to change her drinking behavior.

\section{THE LONG-TERM ROLE OF SOCIAL NETWORKS ON TREATED AND UNTREATED PROBLEM DRINKERS}

\section{Lyndsay N. Ammon, Lee Ann Kaskutas, and Jason Bond}

\section{Introduction}

Higher rates of alcohol abstinence have been associated with attendance at Alcoholics Anonymous (AA) in both treated and untreated populations. For example, Ouimette et al. (1998) reported higher rates of alcohol abstinence among people who attend AA compared with people who do not attend AA at both 12 and 18 months posttreatment. Additionally, Project $\mathrm{MATCH}$ found AA attendance positively predicted abstinence during treatment, at 6 months, 12 months, and 10 years posttreatment (Tonigan et al., 2002, 2003). In an untreated population, attendance at a greater number of AA meetings between baseline and the 1-year follow-up was associated with abstinence at the 1-year follow-up (Timko et al., 1994) and 8 years postbaseline (Moos and Moos, 2004).

Studies have found that changes in social networks help to explain AA's effect on alcohol-related outcomes. For example, a reduction in the number of heavy drinkers and the number who encourage use partially explained the relationship between AA involvement and reduced substance abuse, with over one-third of AA's effect explained by having fewer such prodrinking influences in one's network (Kaskutas et al., 2002). The quality of social networks also has been related to abstinence. Humphreys and Noke (1997) found that those networks based on friendship quality and support for abstinence mediated the relationship between AA involvement and substance abuse. AA participation also has been found to inoculate patients from the negative effects of a heavily drinking social network, but these findings did not emerge until the 3-year follow-up of Project MATCH (Project MATCH Research Group, 1998).

Research regarding the influence of both supportive and negative social networks, and how these related to AA involvement, has not been conducted in treated and untreated problem and dependent drinkers over time. The presentation at RSA had 3 goals: (1) to find whether supportive social networks help explain the association between AA and abstinence over time in a combined sample of treated and untreated problem drinkers; (2) to investigate whether supportive social networks worked with AA attendance in boosting the likelihood of abstinence in this sample; and (3) to evaluate whether AA attendance inoculated these problem drinkers from a network of people who encouraged drinking or drug use.

\section{Methods}

Participants included 926 problem drinkers recruited from 1 of 10 treatment facilities in a Northern California Bay Area County. Trained interviewers, independent from the treatment agencies, conducted in-person interviews. The data were weighted to account for the differences in fieldwork duration across agencies and nonresponse differences in agencies. In addition, 672 untreated problem drinkers were recruited from the same county via telephone interviews using random digit dialing. These untreated participants were recruited if they had not attended chemical dependency treatment in the previous 12 months and if they met problem drinking criteria. In this study problem drinking was defined as a positive response to 2 of the following 3 criteria: (1) drinking 5 or more 
drinks a day monthly (for women drinking 3 or more drinks a day weekly); (2) one or more (8 possible) alcoholrelated social consequences; or (3) one or more dependence symptoms from a checklist of 9 . The 2 samples were reinterviewed $1,3,5$, and 7 years postbaseline, with high response rates $(84,82,79$, and $75 \%$, respectively) in the combined sample.

The outcome variable for all 3 analyses was a dichotomous 30-day alcohol abstinence indicator at follow-up. Analyses for the first 2 aims included a supportive social network independent variable, number of people in one's social network who support cutting down drinking and drug use, along with time and the number of AA meetings attended in the past 12 months. The analyses for the final aim included a negative social network variable, number of people in one's social network who were heavy drinkers or use drugs, in lieu of the positive social network variable. This model also included time and the number of AA meetings attended in the past 12 months.

Generalized estimating equations (GEE) were used to analyze these longitudinal data. In addition, a formal test of mediation was conducted to test aim 1.

\section{Results}

Corresponding to aim 1, supportive social networks mediated the relationship between past 12-month AA meeting attendance and alcohol abstinence (Goodman test for mediation $=2.65, p=0.008$ ), although the change in the coefficient for the relationship between AA meetings and abstinence was modest (a decrease from 0.0149 to $0.0146)$. With regard to aim 2 , a negative interaction between AA meeting attendance and supportive social networks was significant $(p=0.004)$ in the GEE model. Thus, although the odds of abstinence were higher for participants with larger supportive social networks, AA was more effective for those with fewer supportive social networks. However, when time was included in the interaction, a positive association was found $(p<0.001)$, suggesting that over time, AA becomes more effective for participants with supportive social networks (Fig. 3).

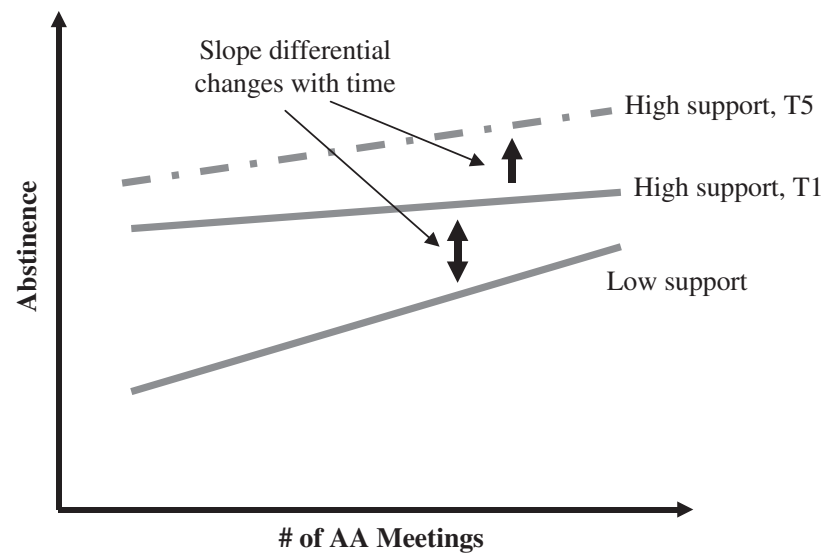

Fig. 3. Moderation: supportive social networks.

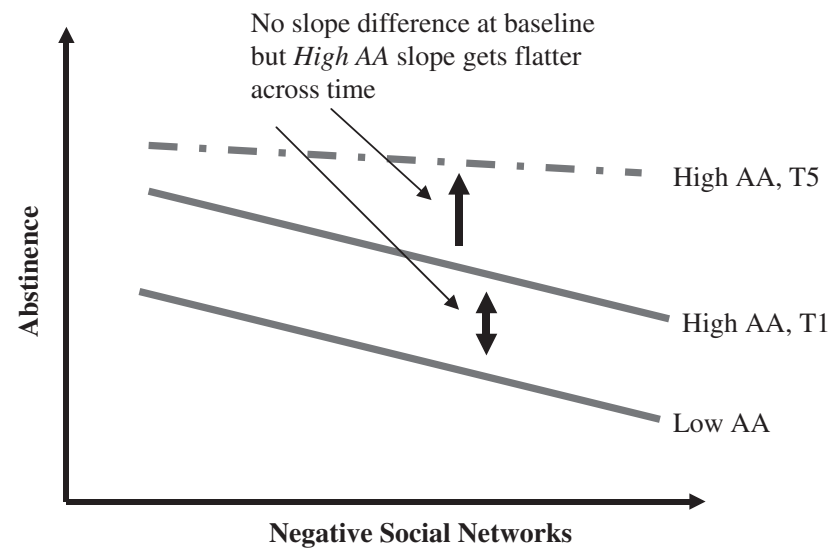

Fig. 4. Moderation: negative social networks.

Finally, regarding aim 3 (the inoculation hypothesis), the interaction between AA meetings and negative social networks was not significant $(p=0.858)$. Yet, when time was added to the interaction of AA meetings and negative social networks, a positive significant association was found $(p<0.020)$. These findings suggest that attending more AA meetings inoculates people with a large network of heavy drinking people, an effect that is more evident over time (Fig. 4).

\section{Discussion}

Our finding that supportive social networks partially explain the influence of AA meeting attendance's effect on abstinence over time supports previous findings of the effect of supportive social networks. These results add to the literature in a sample that includes both treated and untreated problem and dependent drinkers across a period of 7 years.

Supportive social networks also were found to influence the relationship between AA attendance and alcohol abstinence. We found that AA appears to be more effective for those with a low amount of supportive social support in the period shortly after treatment. This suggests that participants with low levels of positive social support should be targeted to continue their AA attendance over time.

Finally, AA meetings were found to help inoculate or protect participants from the negative influences of social networks with large numbers of heavy drinkers. However, this effect was found only when interacted with time, thus suggesting that inoculation only occurs after several years have passed. These findings support Project MATCH's previous findings and add to the literature these results in a sample of treated and untreated problem and dependent drinkers. They also suggest that problem and dependent drinkers with high levels of negative support should maintain their AA attendance over time to limit the impact of the negative social influences they may run into later in life. Last, these results highlight the value of repeated interviews across long follow-up periods, so that long-term effects can be discerned. 
ENVIRONMENTAL INFLUENCES ON LIKELIHOOD OF ALCOHOL TREATMENT-SEEKING BEHAVIOR AMONG YOUNG ADULTS IN THE MILITARY

Genevieve M. Ames, Carol B. Cunradi, and Roland S. Moore

\section{Introduction}

Military personnel have the option of seeking treatment for an alcohol-related problem from either a military or a civilian treatment program. Past research has shown that most service members seek treatment from military treatment programs (Bray et al., 2003), which include peer Drug and Alcohol Program Advisors (DAPA). Despite the increase in heavy drinking among military personnel over the past 4 years (Bray et al., 2003), there is a paucity of research on the factors associated with alcohol treatment-seeking behavior among military personnel. The purpose of the current study is to assess the environmental influence of occupational factors (e.g., supervisor encouragement for going to DAPA, workplace social support for going to DAPA, belief that going to DAPA will harm one's career, and belief in DAPA efficacy) on the likelihood of alcohol treatment seeking behavior from either a military or civilian counselor among a cohort of young adults in the Navy. Additionally, this study examines beliefs about perceived consequences of alcohol treatment-seeking behavior within the military. These data are part of a larger prevention-oriented study on the interaction of individual and environmental factors on drinking among young adults in the US Navy (Ames et al., 2002; Cunradi et al., 2005).

\section{Methods}

This study used a mixed methods approach that integrates qualitative (ethnography) and quantitative (survey research) methods. For the ethnography, naturalistic observations and 50 tape-recorded, semistructured confidential interviews were conducted with young sailors and officers, as well as with supervisors and medical personnel who work closely with young sailors, on Navy bases in the Atlantic and Pacific Fleets and abroad. Using the qualitative text management program ATLAS.ti (Muhr, 2004), staff anthropologists coded the transcripts of these interviews for recurring themes related to study objectives. Relative salience of themes was compared between ranks, ethnic groups, and work settings.

For the survey, confidential self-administered questionnaires were voluntarily completed by 713 enlisted Navy personnel $(69 \%$ male; $48 \%$ nonwhite; mean age 20.9 years) and 419 officers ( $89 \%$ male; $19 \%$ nonwhite; mean age 27.5 years). Information was obtained on alcohol patterns and problems, demographics (gender, age, rank, race/ethnicity), beliefs about supervisor support for DAPA alcohol treatment-seeking behavior, likelihood of alcohol treatment-seeking behavior from a military or civilian counselor, and perceived consequences of seeking help from the DAPA (i.e., one's chances for promotion would be hurt, or one would be involuntarily discharged or separated from the Navy). Separate analyses were conducted among enlisted personnel and officers.

\section{Results and Discussion}

Among enlisted personnel, 25\% reported that they were likely to go to the DAPA only if they thought they had a drinking problem; $10 \%$ reported that they were likely to go to the civilian alcohol counselor only; $40 \%$ reported that they were likely to go to either the DAPA or the civilian alcohol counselor; and 25\% reported that they were likely to go to neither. Among officers, $25 \%$ reported that they were likely to go to the DAPA only if they thought they had a drinking problem; $21 \%$ reported that they were likely to go to the civilian alcohol counselor only; $31 \%$ reported that they were likely to go to either the DAPA or the civilian alcohol counselor; and $23 \%$ reported that they were likely to go to neither.

Multinomial logistic regression models were developed to simultaneously evaluate the influence of environmental factors on the odds of reporting likelihood of alcohol treatment seeking with (1) the DAPA only, (2) the civilian alcohol counselor only, or (3) either the DAPA or the civilian alcohol counselor, compared with those reporting they were unlikely to go to either the DAPA or civilian alcohol counselor. Overall, the results indicated that environmental factors (e.g., supervisor encouragement for going to the DAPA, workplace social support for going to the DAPA, belief that going to the DAPA will harm one's career, and belief in DAPA efficacy) were significantly associated among enlisted personnel and officers with reported likelihood of going to either the DAPA or civilian alcohol counselor. In terms of nonenvironmental factors, frequent heavy drinking was significantly associated with lower odds of reported likelihood of going to either DAPA or civilian alcohol counselor among enlisted personnel only. Nonwhite race/ethnicity was associated with higher odds of reported likelihood of going to the DAPA and/or civilian alcohol counselor.

The results of the ethnography revealed ambivalence among supervisors on whether to enact formal treatment referral policy or to use a "wait and see" informal policy when confronted with problem drinkers in the military workplace. Both sailors and officers seemed evenly divided as to whether or not going to alcohol treatment would be harmful to one's military career. Most believed that selfreferral to alcohol treatment would not be harmful to one's career, but that mandatory referral by one's supervisor would have a detrimental impact on one's chances of promotion. Finally, most junior enlisted interviewed believed that higher-ranked personnel received more leniency in the face of their workplace problem drinking behavior, although higher-ranking enlisted and officers 
believed that a serious alcohol incident such as a DUI would effectively end their military careers.

In summary, the present study provides support for a model of treatment seeking that includes environmental factors in the workplace, including peer support as a complement to supervisor referral. The study results suggest that coworkers and supervisors represent systems of support that could be brought into play by treatment providers to aid problem drinkers in the arduous task of asking for help. Belief in treatment efficacy appears to have a strong effect on the use of available alcohol treatment services. Involvement of coworkers and supervisors enhances these positive beliefs. Lastly, our findings were consistent with previous research (i.e., Delaney et al., 1998) that demonstrated similar environmental influences on alcohol treatment-seeking behavior among employees in a unionized heavy machinery manufacturing plant. Understanding the role of environmental influences, such as supervisor support for treatment-seeking, on the likelihood of alcohol treatment-seeking behavior can aid worksite efforts to encourage those with alcohol-related problems to begin the treatment-seeking process.

\section{DISCUSSION}

\section{Richard Longabaugh}

Alcohol use disorders are described as biopsychosocial disorders, where the causes of the disorder are the result of the interaction among biological, psychological, and social factors, and effective interventions may involve biological, psychological, and/or interpersonal mechanisms of action. Despite this recognition, historically the focus for treating AUDs has been on psychological and, more recently, biological processes. Social influences have been understudied. Nevertheless, the excellent presentations in this symposium nicely exemplify the considerable progress that has been made over the past 25 years in examining and understanding the social processes that affect and are affected by AUDs.

To put today's presentations in historical context, early studies of the effect of social influences on AUD would sometimes use presence of a spouse as a surrogate for social support. As interest grew in understanding social influences, the construct of social support became a focus of study in its own right. But social support was viewed as a singular construct, with the initial hypothesis that those who had it would fare better than those who did not. Results from these early studies were quite equivocal and inconsistent. It then became clear that if social support was to be a useful explanatory variable, the construct would need to be clarified and differentiated: For example: What kind of support? Support for what? Support from whom? This realization led to research where alcohol-specific support was differentiated from general emotional and/or instrumental support (Beattie and Longabaugh, 1999; Beattie et al., 1993) and where the sources of this support were identified and studied in their separate domains: work, spouse and family, as well as the entire social network. The differentiation of general from alcohol-specific support proved useful, and provided evidence that alcohol-specific support was more closely related to drinking behavior than was general social support, but that each could moderate the strength of the relationship of the other to alcohol use (Beattie and Longabaugh, 1997). Despite this basic understanding, some investigators continue to conduct investigations where general support is not differentiated from alcohol-specific support and perhaps not coincidentally, continue to find equivocal results (e.g., COGA).

In contrast, today's presentations have greatly elaborated on these basic understandings and extend our knowledge base, as well as identify interesting unexpected findings that are tantalizing.

Ammon et al. perhaps surprise us the most by testing 3 hypotheses well informed by the existing knowledge base concerning the long-term role of social networks on drinkers. What is surprising, in this instance, is that all 3 hypotheses are supported. Focusing on mechanisms of change, they find that supportive social networks in part explain why AA attendance helps abstinence. They also find that AA and supportive social networks moderate one another's effect on abstinence. And finally, AA inoculates participants from a natural social network that is supportive of drinking, consistent with Project MATCH results (Longabaugh et al., 1998). These findings are entirely consistent with a conceptual framework that differentiates the effects of a natural social network from the AA network and how each impacts the other. Consistent with other AA research, the social influence of AA as a mechanism of change is exemplified. The implications of this research are that AA's impact can be decomposed and that the effects of entire social networks on drinkers can be moderated by AA participation.

One part of this natural social network is the social system of work. Ames et al.'s study of environmental influences on treatment seeking behavior in the military uses a combination of quantitative and qualitative data to present the case that treatment seeking includes both coworker and supervisor support systems as factors that affect the problem drinker's probability of asking for help. Her findings are quite consistent with expectations. Belief in treatment efficacy has a strong effect on use of available treatment, and coworker and supervisor involvements enhance these positive beliefs. This fits well into the existing knowledge base. A mystery left to be solved is the finding that nonwhite race/ethnicity is associated with a higher probability of reported likelihood of going for counseling. "Ethnicity," like gender, is a "trait" measure that calls for dynamic processes that will explain the relationship of these "traits" to the remediation of AUDs. While we can develop numerous speculations for such relationships, these speculations need to be fully articulated and tested. 
Another part of the natural social network is, of course, the family. McCrady and Epstein's study of family influences on women drinkers is novel. Their most interesting finding is that the impact of women, especially sisters, is substantial. In contrast, the influence of males in the family is negligible. This is a surprise that should open up a whole new line of inquiry. Also clarifying the existing knowledge base, it appears as though the number of family members, rather than the percentage, and not supporting drinking as opposed to supporting drinking, are the more important sources of influence on drinking. The social investment hypothesis of Longabaugh et al. (1993), as indexed by the "importance" of family members, was not supported. This may be a failure of theory or alternatively may speak to the inadequate capture of investment by a single question regarding "importance." The initial test of this hypothesis that was supported (Longabaugh et al., 1993) included a more extensive measure of "social investment." It may be premature to reject the investment hypothesis, which otherwise commands considerable theoretical plausibility. On the other hand, the family is a social system that may have unique characteristics that as yet have not been factored in to adequately describe a person's social network. This is a question in need of further examination.

Molina et al.'s examination of moderating and mediating factors among ADHD youth at risk of alcoholism is guided by Sher's model of alcoholism vulnerability. This study is state of the art in its look at mechanisms of change and is exactly the kind of research needed. To sample their findings, the relationship between childhood ADHD (a "person" variable) and adolescent substance use (also a "person" variable) is moderated by peer substance use (an interpersonal, social influence variable). The frequency of heavy drinking by an adolescent 1 year later, predicted by the frequency of parental drinking, is mediated by the adolescent's positive alcohol expectancies. This focus on explanatory interpersonal variables helps to "connect the dots." Yet the finding that negative alcohol expectancies predict less frequent heavy drinking only for control offspring indicates that ADHD is a moderator of this relationship. This leaves us with the question, what is it about ADHD personality that breaks this causal chain? Is it that expectancy is a trait-like variable that is suppressed by the features of ADHD? And lastly, the finding that density of alcohol problems in the family is associated with child behavior only in families with lower quality parenting practices again provides an expected result as to the mechanism of action in this relationship. Examining factors that affect intergeneration influences expands the contextual framework that is needed to understand the etiology of alcohol problems.

Zucker also examines intergeneration effects, but adds to the complexity of the model by testing the moderating influences of grandparent and parent aggression and conduct problems. And indeed aggression and conduct problems moderate these cross generation effects. A take home message is that person variables need to be consider- ed when attempting to trace the effect of alcohol disorders on subsequent generations. This unwanted complexity nevertheless brings us to model constructions that more closely approximate the complexity of the phenomena we are trying to understand.

Zucker also takes us in the other direction, from the broader context in which to understand AUDs to the microtext for understanding the process of how these effects occur. By studying the interactions of marital partners, some with, and some without accompanying ASPD, he identifies negative marital interaction processes that become routinized and regarded as "characterological" or "diagnostic." He finds that couples with an ASPD alcoholic husband have more hostile behaviors than those couples where the husband is not an ASPD. He also finds that couples where both the husband and wife have AUDs have more positive behaviors than couples with a nonalcoholic wife. These findings support the status of ASPD as a moderator variable and also of congruence in marital AUDs as a facilitator of positive interactions in the relationship. Again, findings such of these go beyond the simpler associations that we have tried to use as sufficient for an explanatory framework in the past. Process studies such as Zucker's will help us to begin to explain more of the variance in etiology and prognosis of AUDs.

In summary, the study of social influences on AUDs has progressed remarkably well. A part of this progress is attributable to the recognition that more complex explanatory models are not only needed, but must be tested. Successful studies such as these bring us significantly closer to understanding the reciprocity of social influence and AUD and the personal, generation and social contextual variables that affect the strength of these relationships. The presenters are to be congratulated on their contributions to this growing body of knowledge.

\section{REFERENCES}

Ames GM, Cunradi C, Moore RS (2002) Alcohol, tobacco, and drug use among young adults prior to entering the military. Prev Sci 3:135-144. Ames GM, Cunradi CB (2004/2005) Alcohol Use and Preventing Alcohol-Related Problems among Young Adults in the Military. Alcohol Research and Health 28 (4): 252-257.

Babor TF (1996) The classification of alcoholics: typology theories from the 19th century to the present. Alcohol Health Res World 20:6-17.

Bagwell CL, Molina BSG, Pelham WE, Hoza B (2001) Attention-deficit hyperactivity disorder and problems in peer relations: predictions from childhood to adolescence. J Am Acad Child Adolesc Psychiatry 40:1285-1292.

Beattie M, Longabaugh R (1997) Interpersonal factors and post-treatment drinking and subject well-being. Addiction 92:1507-1521.

Beattie MC, Longabaugh R (1999) General and alcohol-specific social support following treatment. Addict Behav 24:593-606.

Beattie MC, Longabaugh R, Elliott G, Stout RL, Fava J, Noel N (1993) Effect of the social environment on alcohol involvement and subjective well-being prior to alcoholism treatment. J Stud Alcohol 54:283-296.

Beckman LJ, Amaro H (1986) Personal and social difficulties faced by women and men entering alcoholism treatment. J Stud Alcohol 47:135-145. 
Bray RM, Hourani LL, Rae KL, Dever JA, Brown JM, Vincus AA, Pemberton MR, Marsden ME, Faukner DL, Vandermaas-Peeler R (2003) 2002 Department of Defense Survey of Health Related Behaviors Among Military Personnel. Report RTI/7841/006-FR, Research Triangle International.

Chassin L, Flora DB, King KM (2004) Trajectories of alcohol and drug use and dependence from adolescence to adulthood: the effects of familial alcoholism and personality. J Abnorm Psychol 113:483-498.

Cunradi CB, Moore R, Killoran M, Ames G (2005) Survey nonresponse bias among young adults: the role of alcohol, tobacco and drugs. Subst Use Misuse 40:171-185.

Delaney W, Grube JW, Ames GM (1998) Predicting likelihood of seeking help through the employee assistance program among salaried and union hourly employees. Addiction 93:399-410.

Dishion TJ, McMahon RJ (1998) Parental monitoring and the prevalence of child and adolescent problem behavior: a conceptual and empirical formulation. Clin Child Fam Psychol Rev 1:61-75.

Faden VB, Day NL, Windle M, Windle R, Grube JW, Molina BS, Pelham WE Jr., Gnagy EM, Wilson TK, Jackson KM, Sher KJ (2004) Collecting longitudinal data through childhood, adolescence, and young adulthood: methodological challenges. Alcohol Clin Exp Res 28:330-340

Floyd FJ, Cranford JA, Klotz-Daugherty M, Fitzgerald HE, Zucker RA (2006) Marital interaction in alcoholic and nonalcoholic couples: alcoholic subtype variations and wives' alcoholism status. J Abnorm Psychol 115:121-130.

Fuller BE, Chermack ST, Cruise KA, Kirsch E, Fitzgerald HE, Zucker RA (2003) Predictors of aggression across three generations among sons of alcoholics: relationships involving grandparental and parental alcoholism, child aggression, marital aggression and parenting practices. J Stud Alcohol 64:472-483.

Gottman JM (1994) What Predicts Divorce? The Relationship between Marital Processes and Marital Outcomes. Erlbaum, Hillsdale, NJ.

Humphreys K, Moos RH, Cohen C (1997) Social and community resources and long-term recovery from treated and untreated alcoholism. J Stud Alcohol 58:231-238.

Humphreys K, Noke JM (1997) The influence of post treatment mutual help group participation on the friendship networks of substance abuse patients. Am J Commun Psychol 25:1-16.

Kaskutas LA, Bond J, Humphreys K (2002) Social networks as mediators of the effect of Alcoholics Anonymous. Addiction 97:891-900.

Leonard KE, Roberts LJ (1998) The effects of alcohol on the marital interactions of aggressive and nonaggressive husbands and their wives. J Abnorm Psychol 10:602-615.

Longabaugh R, Beattie M (1985) Optimizing the cost effectiveness of treatment for alcohol abusers, in Future Directions in Alcohol Abuse Treatment Research (McCrady BS, Noel NE, Nirenberg TD eds), pp 104-136. NIAAA Research Monograph \#15, DHHS Pub. No. 9ADM) 85-1322. U.S. Government Printing Office, Washington, DC.

Longabaugh R, Beattie M, Noel N, Stout R, Malloy P (1993) The effect of social investment on treatment outcome. J Stud Alcohol 54:465-478.

Longabaugh R, Wirtz PW, Zweben A, Stout RL (1998) Network support for drinking, Alcoholics Anonymous and long-term matching effects. Addiction 93:1313-1333.

Marshal MP, Molina BSG, Pelham WE (2003) Childhood ADHD and adolescent substance use: an examination of deviant peer group affiliation as a risk factor. Psychol Addict Behav 17:293-302.

McAweeney MJ, Zucker RA, Fitzgerald HE, Puttler LI, Wong MM (2005) Individual and partner predictors of recovery from alcohol use disorder over a nine-year interval: findings from a community sample of alcoholic married men. J Stud Alcohol 66:220-228.

McCrady BS (2004) To have but one true friend: implications for practice of research on alcohol use disorders and social networks. Psychol Addict Behav 18:113-121.

Molina BSG, Marshal MP, Pelham WE Jr, Wirth RJ (2005) Coping skills and parent support mediate the association between childhood ADHD and adolescent cigarette use. J Pediatr Psychol 30:345-357.
Molina BSG, Pelham WE (2003) Childhood predictors of adolescent substance use in a longitudinal study of children with ADHD. J Abnorm Psychol 112:497-507.

Molina BSG, Pelham WE, Gnagy EM, Thompson AL, Marshal MP. ADHD risk for heavy drinking and alcohol use disorder are agespecific. (under review).

Moos RH, Moos BS (2004) Long-term influence of duration and frequency of participation in Alcoholics Anonymous on individuals with alcohol use disorders. J Consult Clin Psychol 72:81-90.

Muhr T (2004) ATLAS.ti 5.0 [Qualitative Text Analytical Software]. ATLAS.ti Scientific Software Development GmbH, Berlin.

Ouimette PC, Moos RH, Finney JW (1998) Influence of outpatient treatment and 12-step group involvement on one-year substance abuse treatment outcomes. J Stud Alcohol 59:513-522.

Pelham WE, Bender ME (1982) Peer relationships in hyperactive children: description and treatment, in Advances in Learning and Behavioral Disabilities (Bailer KGI ed) 1, pp 366-436. JAI Press, Greenwich, CT.

Pelham WE, Gnagy EM, Greenslade KE, Milich R (1992) Teacher ratings of DSM-III-R symptoms for the disruptive behavior disorders. J Am Acad Child Adolesc Psychiatry 31:210-218.

Pelham WE, Hoza B (1996) Intensive treatment: a summer treatment program for children with ADHD, in Psychosocial Treatments for Child and Adolescent Disorders: Empirically Based Strategies for Clinical Practice (Hibbs ED, Jensen PS eds), pp 311-340. American Psychological Association Press, New York.

Pelham WE, Lang AR, Atkeson B, Murphy DA, Gnagy EM, Greiner AR, Vodde-Hamilton M, Greenslade KE (1998a) Effects of deviant child behavior on parental alcohol consumption. Am J Addict 7:103-114.

Pelham WE, Wheeler T, Chronis A (1998b) Empirically supported psychosocial treatments for ADHD. J Clin Child Psychol 27: 190-205.

Project MATCH Research Group (1998) Matching alcoholism treatments to client heterogeneity: Project MATCH three-year drinking outcomes. Alcohol Clin Exp Res 22:1300-1311.

Room R, Greenfield TK, Weisner C (1991) People who might have liked you to drink less: changing responses to drinking by U.S. family members and friends, 1979-1990. Contemp Drug Prob 18:573-595.

Sher KJ (1991) Models of vulnerability, in Children of Alcoholics: A Critical Appraisal of Theory and Research. The University of Chicago Press, Chicago.

Sher KJ, Trull TJ (1994) Personality and disinhibitory psychopathology: alcoholism and antisocial personality disorder. J Abnorm Psychol 103:92-102.

Timko C, Moos RH, Finney JW, Moos BS (1994) Outcome of treatment for alcohol abuse and involvement in Alcoholics Anonymous among previously untreated problem drinkers. J Ment Health Adm 21: $145-160$.

Tonigan JS, Connors GJ, Miller WR (2003) Participation and involvement in Alcoholics Anonymous, in Treatment Matching in Alcoholism (Babor TF, Del Boca FK eds), pp 184-204. Cambridge University Press, Cambridge.

Tonigan JS, Miller WR, Carroll L (2002) Internalization of AA practices 10 -years after treatment: Project MATCH preliminary findings. Presentation abstract, research society on alcoholism. Alcohol Clin Exp Res 26:42A.

Windle M (1997) Mate similarity, heavy substance use and family history of problem drinking among young adult women. J Stud Alcohol 58:573-580

Zucker RA, Fitzgerald HE, Refior SK, Puttler LI, Pallas DM, Ellis DA (2000) The clinical and social ecology of childhood for children of alcoholics: description of a study and implications for a differentiated social policy, in Children of Addiction: Research, Health and Policy Issues (Fitzgerald HE, Lester BE, Zuckerman BS eds), pp 109-141. Routledge Falmer, New York.

Zucker RA, Wong MM (2005) Prevention for childern of alcoholics and other high risk group in, Recent developments in alcoholism: Volume 17. Alcohol problems in adolescents and young adults (Galanter M ed), pp. 299-320. Kluwer Academic/Plenum Publishers, New York. 